Pacific Journal of Mathematic 


\section{BASE CHANGE LIFTING AND GALOIS INVARIANCE}

JOE REPKA

Let $G$ be a quasi-split connected reductive group defined over the reals. Every irreducible representation $\pi$ of $G_{R}$ has a base change lifting $\Pi$, a representation of $G_{C}$, such that $\Pi$ is equivalent to its conjugate $\Pi^{o}$. We prove that if $G=\mathrm{GL}(n)$, every $\Pi$ which is equivalent to $\Pi^{\sigma}$ is the lifting of some $\pi$, but show by examples that this is not always true for general $G$. Finally we discuss the analogous global question and show that there are global cusp forms on PGL(2) which are Galois invariant but not liftings.

o. The relationship between a representation $\pi$ of $G_{R}$ and its base change lifting $\Pi$ has been studied for various groups $G$ by several authors, starting with Langlands [6], whose work on the global problem for GL(2) includes the archimedean case, almost in passing. It is expected that the characters of $\pi$ and $\Pi$ are related, in a specific way, via the norm map, at least when $\pi$ is tempered. This relation has in fact been proved by Shintani [8] for $G L(2, R)$, by Clozel [2] for representations of $\mathrm{GL}(n, \boldsymbol{R})$ induced from unramified quasicharacters of a minimal parabolic subgroup, and in a forthcoming paper by the present author [7] for arbitrary tempered irreducible representations of $\mathrm{GL}(n, \boldsymbol{R})$.

In this paper we address the question of whether a given representation $\Pi$ of $G_{c}$ is the lifting of some $\pi$. We first interpret the action of the Galois group of $\boldsymbol{C} / \boldsymbol{R}$ on representations of $G_{\boldsymbol{C}}$ in terms of the Langlands classification for these representations. Then we use our results to study liftings. We shall work directly with the $L$-homomorphisms corresponding to $\pi$ and $\Pi$, rather than the representations themselves, and do not here broach the more difficult question of the relationship between $\pi$ and $I$.

We use the notations and terminology of [5], except that, following [1], we write ${ }^{L} G^{\circ}$ for the dual group. Thus ${ }^{L} G^{\circ}$ is a connected complex Lie group. Since $G$ is defined over $R$, there is an action of $\Gamma=\operatorname{Gal}(\boldsymbol{C} / \boldsymbol{R})$ on ${ }^{L} G^{\circ}$, and if $\sigma$ is the nontrivial element of $\Gamma$ we denote this action by $g \mapsto \sigma \cdot g$. The Weil group $W_{R}$ also acts on ${ }^{L} G^{\circ}$, and we form the real dual group ${ }^{L} G_{R}={ }^{L} G^{\circ} \rtimes W_{R}$ and the complex dual group ${ }^{L} G_{C}={ }^{L} G^{\circ} \times W_{c}$.

1. An irreducible representation $\Pi$ of $G_{C}$ is associated to a (class of) $L$-homomorphisms $\Phi: W_{\boldsymbol{c}} \rightarrow{ }^{L} G_{\boldsymbol{c}}$ (see [5]). We define the 
representation $\Pi^{\sigma}$ of $G_{c}$ by $\Pi^{\sigma}(g)=\Pi\left(g^{\sigma}\right)$, where $g^{\sigma}$ is just the complex conjugate of $g \in G_{c}$. Then $\Pi^{\sigma}$ is associated to a (class of) $L$-homomorphisms $\Phi^{\sigma}: W_{c} \rightarrow{ }^{L} G_{c}$, which we now describe. As usual, identify $W_{c}=C^{\times}$, and write $\Phi(z)=a(z) \times z \in{ }^{L} G^{\circ} \times W_{c}$. Then

Proposition 1. A representative of $\Phi^{\sigma}$ is

$$
\Phi^{\sigma}(\boldsymbol{z})=[\sigma \cdot a(\bar{z})] \times \boldsymbol{z} .
$$

Proof. We may assume that the image of $\Phi$ is contained in a maximal torus ${ }^{L} T^{\circ} \times W_{c}$ of ${ }^{L} G_{C}$ such that $\sigma\left({ }^{L} T^{\circ}\right)={ }^{L} T^{\circ}$. Following [5], form $L, L^{\wedge}$ as usual. There is an action of $\Gamma$ on $L$, given by

$$
\left.\sigma(\lambda)\left(t^{\sigma}\right)=\overline{\lambda(t}\right), \quad\left(\lambda \in L, t \in T_{\mathbf{c}}\right) .
$$

It induces a dual action on $L^{\wedge}$, which is compatible with the action on ${ }^{L} G^{\circ}$.

Next we restrict scalars; i.e., find groups $S, H$ so that $S_{R} \cong T_{c}$, $H_{R} \cong G_{C}$. Then ${ }^{L} S^{\circ} \cong{ }^{L} T^{\circ} \times{ }^{L} T^{\circ}$ and ${ }^{L} H^{\circ}={ }^{L} G^{\circ} \times{ }^{L} G^{\circ} ; \Gamma$ acts by transposition:

$$
\sigma^{\prime}\left(g_{1}, g_{2}\right)=\left(g_{2}, g_{1}\right) \text {. }
$$

Corresponding to $S$, form $L^{\prime} \cong L \times L, L^{\prime \wedge} \cong L^{\wedge} \times L^{\wedge}$, with the natural duality: $\left\langle\left(\lambda_{1}, \lambda_{2}\right),\left(\hat{\lambda_{1}}, \lambda_{2}\right)\right\rangle=\left\langle\lambda_{1}, \lambda_{1}\right\rangle+\left\langle\lambda_{2}, \lambda_{2}\right\rangle$.

From the action (1) of $\sigma$ on $L$ we get an action on $L^{\prime}$ by $\sigma\left(\lambda^{\prime}\right)=$ $\sigma\left(\lambda_{1}, \lambda_{2}\right)=\left(\sigma \lambda_{1}, \sigma \lambda_{2}\right)$. If $t \in T_{c}, \lambda^{\prime} \in L^{\prime}$, this action satisfies the analogue of (1), namely

$$
\sigma\left(\lambda^{\prime}\right)\left(t^{\sigma}\right)=\overline{\lambda^{\prime}(t)}
$$

By duality, there is an action on $L^{\prime \wedge}=L^{\wedge} \times L^{\wedge}$ and also on ${ }^{L} S^{\circ}$ and ${ }^{L} H^{\circ}$; both actions are componentwise: $\sigma\left(g_{1}, g_{2}\right)=\left(\sigma \cdot g_{1}, \sigma \cdot g_{2}\right)$, if $\left(g_{1}, g_{2}\right) \in{ }^{L} H^{\circ} \cong{ }^{L} G^{\circ} \times{ }^{L} G^{\circ}$.

Now from the action $\sigma^{\prime}$ given by (2), we get another action $\sigma^{\prime}$ on $L^{\prime \wedge}$, and by duality on $L^{\prime}$, the latter given by $\sigma^{\prime}\left(\lambda_{1}, \lambda_{2}\right)=\left(\lambda_{2}, \lambda_{1}\right)$. If $\lambda^{\prime} \in L^{\prime}, t=\left(t_{1}, \cdots, t_{n}\right) \in T_{\boldsymbol{c}} \cong\left(\boldsymbol{C}^{\times}\right)^{n}$, and $\bar{t}=\left(\bar{t}_{1}, \cdots, \bar{t}_{n}\right)$, then this action satisfies

$$
\sigma^{\prime}\left(\lambda^{\prime}\right)(t)=\lambda^{\prime}(\bar{t})=\overline{\lambda^{\prime}(t)} .
$$

The automorphism $g \mapsto g^{\sigma}$ of $G_{C}$, regarded as the real group $H_{R}$, induces an automorphism of the (real) dual group ${ }^{L} H^{\circ}$ (and hence of $\left.{ }^{L} H_{R}\right)$. We wish to calculate this dual automorphism, ${ }^{L} \sigma$.

If $\lambda^{\prime} \in L^{\prime}, t \in T_{c}$, we find, using (3) and (4), that $\sigma \sigma^{\prime}\left(\lambda^{\prime}\right)\left(t^{\sigma}\right)=$ $\overline{\sigma^{\prime}\left(\lambda^{\prime}\right)(t)}=\lambda^{\prime}(t)$. Thus $\sigma \sigma^{\prime}$ is the action on $L$ dual to $t \mapsto t^{\sigma}$ on $T_{c}$. This allows us to calculate the action on ${ }^{L} H^{\circ}$ (and hence ${ }^{L} H_{R}$ ), 
namely

$$
{ }^{L} \sigma\left(g_{1}, g_{2}\right)=\sigma \sigma^{\prime}\left(g_{1}, g_{2}\right)=\left(\sigma g_{2}, \sigma g_{1}\right) .
$$

Now given our $\Phi: W_{c} \rightarrow{ }^{L} G_{c}$, we restrict scalars and find the corresponding $\phi: W_{R} \rightarrow{ }^{L} H_{R}$ (cf. [5], p. 13). Composing ${ }^{L} \sigma$ with $\phi$, we obtain $\phi^{\sigma}$. Reversing the restriction of scalars process, we obtain $\Phi^{o}$.

Explicitly, following [5], pp. 12-13, we let $V=\{(1,1),(1, \sigma)\}$ and find $\phi(z, 1)=(a(z), a(\bar{z})) \times(z, 1)$, and $\phi^{\sigma}(z, 1)=(\sigma \cdot a(\bar{z}), \sigma \cdot a(z)) \times(z, 1)$. From this we see that $\Phi^{\sigma}(z)=\sigma \cdot a(\bar{z}) \times z$.

REMARK. We had to restrict scalars because the automorphism $\sigma$ of $G_{C}$ is not defined over $C$, though the corresponding automorphism of $H_{\boldsymbol{R}}$ is defined over $\boldsymbol{R}$.

2. If $\phi: W_{R} \rightarrow{ }^{L} G_{R}$ is an $L$-homomorphism, its restriction to the subgroup $W_{c}$ has its image contained in ${ }^{L} G_{c}$, so is an $L$-homomorphism $\Phi: W_{c} \rightarrow{ }^{L} G_{c}$. In this situation we say $\Phi$ is a "lift" of $\phi$. It is easily seen that for such a $\Phi$, we have $\Phi^{\sigma} \sim \Phi$, i.e., $\Phi^{\sigma}=\operatorname{Ad}(g) \Phi$, for some $g \in{ }^{L} G^{\circ}$ (in fact, if $\phi(1, \sigma)=h \times(1, \sigma)$, then $g=\sigma \cdot h$ will work). The question at hand is the converse: suppose an $L$-homomorphism $\Phi$ satisfies $\Phi^{\sigma} \sim \Phi$. Must $\Phi$ be the lift of some $\phi$ ? We shall see that the answer is "sometimes".

Given $\Phi$ with $\Phi^{\sigma} \sim \Phi$, we must try to extend $\Phi$ to an $L$-homomorphism $\phi: W_{R} \rightarrow{ }^{L} G_{R}$. The difficulty is to define $\phi(1, \sigma)$ so that

$$
\phi(1, \sigma) \Phi(z) \dot{\phi}(1, \sigma)^{-1}=\Phi(\bar{z})
$$

and

$$
\phi(1, \sigma)^{2}=\Phi(-1) .
$$

In light of (1), a natural first choice for $\phi(1, \sigma)$ is $\sigma \cdot g \times(1, \sigma)$, where $g \in{ }^{L} G^{\circ}$ is an element with $\Phi^{\sigma}=\operatorname{Ad}(g) \Phi$, but we may need to modify this choice to satisfy (2).

At this point, two examples are in order.

ExAmple 1. Let $G=\operatorname{PGL}(2)$, so ${ }^{L} G^{\circ}=\mathrm{SL}(2, C)$. Define $\Phi: W_{\boldsymbol{c}} \rightarrow$ ${ }^{L} G^{\circ} \times W_{c}$ by

$$
\Phi(z)=\left(\begin{array}{cc}
\exp \text { in } \arg z & 0 \\
0 & \exp (-\operatorname{in} \arg z)
\end{array}\right) \times z
$$

Then, since the action of $\sigma$ on ${ }^{L} G^{\circ}$ is trivial, $\Phi^{\sigma}(z)=\left(\begin{array}{c}\exp (-\operatorname{in} \arg z) \\ 0\end{array}\right.$ exp in $\arg z) \times z$, and if $g=\left(\begin{array}{rr}0 & 1 \\ -1 & 0\end{array}\right)$ then $\operatorname{Ad}(g) \Phi=\Phi^{\sigma}$, i.e., $\Phi^{\sigma} \sim \Phi$. 
However, $g^{2}=-$ id. If $n$ is odd, we may define $\phi(z, 1)=\Phi(z), \phi(1, \sigma)=$ $g \times(1, \sigma)$, and (1) and (2) will be satisfied. Thus $\phi$ is an $L$-homomorphism $\phi: W_{R} \rightarrow{ }^{L} G_{R}$ and $\left.\phi\right|_{W_{C}}=\Phi$ : i.e., $\Phi$ is the lift of $\phi$. On the other hand, it won't work if $n$ is even, and in fact it is easily checked that no choice of $g$ will satisfy (1) and (2) if $n$ is even. Thus $\Phi^{o} \sim \Phi$ for all $n$, but $\Phi$ is a lift if and only if $n$ is odd.

ExAmple 2. Let $G=\mathrm{SL}(2)$, so ${ }^{L} G^{\circ}=\operatorname{PGL}(2, C)$. Define $\Phi$ by $\Phi(z)=\left(\begin{array}{ccc}\exp \text { in } \arg z & 0 \\ 0 & 1\end{array}\right) \times z$, where by the matrix we understand its image in $\operatorname{PGL}(2, C)$. Then $\Phi^{\circ}(z)=\left(\begin{array}{cc}\exp (-\operatorname{in} \arg z) & 0 \\ 0 & 1\end{array}\right) \times z$. If we let $g=\left(\begin{array}{ll}0 & 1 \\ 1 & 0\end{array}\right)$, then $\operatorname{Ad}(g) \Phi(z)=\left(\begin{array}{lc}1 & 0 \\ 0 & \exp \text { in } \arg z\end{array}\right) \times z=\left(\begin{array}{c}\exp (-\operatorname{in} \arg z) \\ 0\end{array}\right)$ $\left.\begin{array}{l}0 \\ 1\end{array}\right) \times z=\Phi^{\sigma}(z)$, since the matrices are in $\operatorname{PGL}(2, C)$. Thus $\Phi^{\sigma} \sim \Phi$. If $n$ is even we may define $\phi(1, \sigma)=g \times(1, \sigma)$ and (1) and (2) will be satisfied. However, if $n$ is odd, $\Phi(-1)=\left(\begin{array}{rr}-1 & 0 \\ 0 & 1\end{array}\right) \times(-1)$, and it is easily checked that no choice of $g$ will work. Thus $\Phi^{\sigma} \sim \Phi$ for all $n$, but $\Phi$ is a lift if and only if $n$ is even.

3. These examples can be explained, to some extent, in terms of the corresponding representations, as follows. In Example 1, it is convenient to think in terms of GL(2). By composing $\Phi$ with the inclusion $\mathrm{SL}(2, C) \rightarrow \mathrm{GL}(2, C)$ we get an $L$-class and hence a representation of $\mathrm{GL}(2, C)$ and this representation is trivial on the center, so it factors to give a representation of $\operatorname{PGL}(2, C)$. However the representation of $\mathrm{GL}(2, \boldsymbol{C})$ is the lift of a representation of $\mathrm{GL}(2, \boldsymbol{R})$ which is not trivial on the center if $n$ is even (its value at $-i d$ is $-1)$, so does not correspond to a genuine representation of $\operatorname{PGL}(2$, $\boldsymbol{R})$. So we have a representation of $\operatorname{PGL}(2, \boldsymbol{C})$ which is Galois invariant but for which the reasonable corresponding representation of $\operatorname{PGL}(2, \boldsymbol{R})$ does not exist.

In Example 2, for even $n$, the representations $\pi$ of $G_{R}$ which correspond to $\phi$ are the discrete series representations corresponding to the characters $\left(\begin{array}{r}\cos \theta \sin \theta \\ -\sin \theta \cos \theta\end{array}\right) \mapsto \exp \pm i n / 2 \theta$, which are not defined for odd $n$.

It should be noticed that other similar examples are not diffificult to find; it is easy to mimic the construction of Example 1 for $G=\operatorname{PGL}(n)$ or when ${ }^{L} G^{\circ}=\operatorname{Sp}(n, C)$.

4. We now discuss some criteria which will help decide whether $\Phi$ is a lift in certain cases.

Proposition 2. If $G$ is split over $\boldsymbol{R}$ and $\Phi^{\sigma}=\Phi$, then $\Phi$ is a lift. 
Proof. Since $\Phi^{\sigma}=\Phi$, rather than just $\Phi^{\sigma} \sim \Phi$, we may take any $g \in{ }^{L} T^{\circ}$ and set $\phi(1, \sigma)=g \times(1, \sigma)$ to satisfy (1). And to satisfy (2), we need only take $g$ so that $\Phi(-1)=g^{2} \times-1$. (Note that since $G$ is split, $\sigma$ acts trivially.)

Taking a cue from the above proof, we look for cases in which $g$ can be found so that $g(\sigma \cdot g)$ has the right value.

Proposition 3. Suppose $G$ is split and suppose $g \in{ }^{L} G^{\circ}$ is such that

(i ) $\Phi^{\sigma}=\operatorname{Ad}(g) \Phi$

(ii) $g^{2}=1$

(iii) $\left({ }^{L} T^{\circ}\right)^{g}$, the subset of ${ }^{L} T^{\circ}$ fixed by $\operatorname{Ad}(g)$, is connected; then $\Phi$ is a lift.

Proof. Let $\Phi(-1)=a \times-1$, with $a \in\left({ }^{L} T^{\circ}\right)^{g}$. Since square roots exist in a connected complex torus, there exists $c \in\left({ }^{L} T^{\circ}\right)^{g}$ with $c^{2}=a$; let $g^{\prime}=c g$. Then $\Phi^{\sigma}=\operatorname{Ad}\left(g^{\prime}\right) \Phi$ and $\left(g^{\prime}\right)^{2}=c g c g=c g c g^{-1}=c^{2}=a$.

Proposition 2 applies, for example, to the $\phi$ 's which arise as lifts of $\phi$ 's corresponding to principal series representations induced from minimal parabolic subgroups.

5. We are now able to prove that there is no trouble for $\mathrm{GL}(n)$.

TheOREm. If $G=\mathrm{GL}(n)$ then $\Phi$ is a lift if and only if $\Phi^{\sigma} \sim \Phi$.

Proof. We know that lifts are Galois invariant. For the other direction we shall apply Proposition 3; we need to verify that it is possible to find a $g$ satisfying (i), (ii), (iii).

Assume ${ }^{L} T^{\circ}$ is the diagonal torus. Thus $\Phi$ and $\Phi^{\sigma}$ are each specified by an ordered $n$-tuple of quasicharacters of $W_{\boldsymbol{c}}$ (the diagonal entries of the projection of $\Phi$ into ${ }^{L} G^{\circ}=\mathrm{GL}(n, C)$ ). Since $\Phi$ and $\Phi^{\sigma}$ are equivalent (i.e., conjugate by an element of $\operatorname{GL}(n, C)$ ) they must involve the same $n$ quasicharacters. In other words, $\Phi^{\sigma}$ is obtained from $\Phi$ by a permutation of the diagonal entries. So we may choose a $g$ in the normalizer of ${ }^{L} T^{\circ}$ so that $\operatorname{Ad}(g) \Phi=\Phi^{\sigma}$. Moreover, since $\left(\Phi^{\sigma}\right)^{\sigma}=\Phi$, the permutation must be of order 1 or 2, so we may choose $g$ with $g^{2}=1$.

Now $\operatorname{Ad}(g)$ acts on ${ }^{L} T^{\circ}$ as a product of (disjoint) transpositions, so that the fixed set $\left({ }^{L} T^{\circ}\right)^{g}$ consists of all elements with certain pairs of entries equal. Such a set is isomorphic to $\left(\boldsymbol{C}^{\times}\right)^{m}$ for some 
$m \leqq n$, so is certainly connected.

Thus Proposition 3 applies and the theorem is proved.

6. The preceding analysis can be applied in other situations. If, for example, the group $G$ is not quasi-split, then similar considerations apply, with the additional difficulty that a Galois invariant $\Phi$ could be the lift of a $\phi$ which is not "relevant" (examples are easily constructed for $U(2))$.

The work with $L$-homomorphisms can also be done for $p$-adic groups, though in the absence of the classification theorem for representations, it lacks the representation-theoretic interpretation. On the other hand it may serve to suggest examples.

We turn now to the analogous global question: if a global cusp form is Galois invariant, must it be a lift? Of course for GL(2) Langlands ([6]) has shown the answer is yes. It would be very interesting to know the answer for $\operatorname{GL}(n)$-especially in light of our earlier local result for $\mathrm{GL}(n, \boldsymbol{R})$.

The purpose of this section is to show that for PGL(2) the answer is no. The idea is similar to our Example 1 above, especially the representation-theoretic discussion in $\S 3$. We observe that it is possible to find a representation of $\mathrm{GL}(2)$ which is not trivial on the center but whose lift is trivial on the center; the lift factors to a Galois invariant representation of PGL(2) which is not a lift.

Indeed, let $F$ be a number field, $E$ a quadratic extension, $I_{F}$ and $I_{E}$ their respective idéles. Let $\chi$ be the grössencharakter of $I_{F}$ which is trivial on $N_{E / F}\left(I_{E}\right)$ (the existence of $\chi$ is guaranteed by class field theory). Now let $\pi$ be a cusp form on $\mathrm{GL}\left(2, \boldsymbol{A}_{F}\right)$ with central character $\chi$ and whose lift, $\Pi$, is a cusp form on $\operatorname{GL}\left(2, \boldsymbol{A}_{E}\right)$. Then the central character of $\Pi$ is $\chi_{\circ} N_{E / F}$, which, by the definition of $\chi$, is trivial (for these facts about liftings, see [6], pp.1.14-1.15). Thus $\Pi$ factors to give a cuspidal representation $\bar{\Pi}$ of $\operatorname{PGL}\left(2, \boldsymbol{A}_{E}\right)$; $\bar{\Pi}$ is Galois invariant but we shall see it cannot be a lift.

Notice that every cuspidal representation of $\mathrm{PGL}\left(2, \boldsymbol{A}_{F}\right)$ gives rise to a cuspidal representation of $\mathrm{GL}\left(2, \boldsymbol{A}_{F}\right)$ by composition with the natural projection. By [6] we know each such representation has a lift, and as above the lift has trivial central character, so it factors to give a representation of $\operatorname{PGL}\left(2, \boldsymbol{A}_{E}\right)$. Thus every cusp form of PGL $\left(2, \boldsymbol{A}_{F}\right)$ already has a lift in this way, so our $\bar{\Pi}$ cannot be the lift of any of them (note that ([6], p. 1.15) $\Pi$ is the lift of at most two representations $\pi$, and that they have the same (nontrivial) central character $\chi$ ).

For an explicit example, let $F=\boldsymbol{Q}, K=\boldsymbol{Q}(\sqrt{-2})$. We first construct a grössencharakter of $K$, as follows. The field $\boldsymbol{Q}_{2}(\sqrt{-2})$ is a ramified quadratic extension of $\boldsymbol{Q}_{2}$, with prime ideal $\mathfrak{p}=(\sqrt{-2})$. 
The units modulo $1+\mathfrak{p}^{3}$ form a cyclic group of order 4 , generated by $u=1+\sqrt{-2}$. Define a character $\psi_{2}$ of $\boldsymbol{Q}_{2}(\sqrt{-2})^{\times}$, trivial on $1+\mathfrak{p}^{3}$, by $\psi_{2}(u)=i, \psi_{2}(\sqrt{-2})=-1$. The rational prime $p=3$ splits in $K$; in the two copies of $\boldsymbol{Q}_{3}$ which result, the element $\sqrt{\overline{-2}}$ is congruent to $1 \bmod (3)$ in the first and to $2 \bmod (3)$ in the second. Thus for the prime elements in these two localizations we may take $1-\sqrt{-2}$ and $1+\sqrt{-2}$ respectively. We define a character $\psi_{3}$ of the product of these two localizations by

$$
\psi_{3}(a, b)=|a / b|^{i \pi / 2 \log 3} \mathrm{sgn}_{2}(a) \operatorname{sgn}_{-3}(b)
$$

(here $\operatorname{sgn}_{\theta}$ means the character of order two of $\boldsymbol{Q}_{3}^{\times}$which is trivial on the norms from $\left.\boldsymbol{Q}_{3}(\sqrt{\bar{\theta}})^{\times}\right)$. For the infinite prime, define $\psi_{\infty} \equiv 1$.

If $x \in K^{\times}$, we embed $x$ in each localization, and so calculate $\psi_{\infty}(x) \psi_{2}(x) \psi_{3}(x, x)$. We do this for $x=-1, \sqrt{-2}, 1+\sqrt{-2}, 1-\sqrt{-2}$, and in each case the answer is 1. Each of these elements is a unit in every other localization, and since $K$ has class number 1 there is a unique grössencharakter $\psi$ of $K$ which has the above local components at the given places and is unramified at every other place.

We make three remarks. First, if we restrict $\psi$ to the diagonal embedding of the rational idèles $I_{Q}$, we get the grössencharakter associated to the extension $\boldsymbol{Q}(\sqrt{3}) / \boldsymbol{Q}$. To see this, we check it at the primes $2,3, \infty$, and then remark as before that these data determine a unique grössencharakter unramified at the other primes. Second, we remark that the prime $p=19$ splits in $K$, and calculate the corresponding local components of $\psi$. In fact, we are interested in the corresponding Euler factor, which we find is $\left(1+i p^{-s}\right)^{2}$. Third, we remark that is does not factor through the norm $N: I_{K} \rightarrow I_{Q}$; consider the idèle which is -1 at the two places lying over 3 and 1 elsewhere. Its norm is the trivial idèle but $\psi$ of it is -1 .

Given our grössencharakter $\psi$ of $K$ which does not factor through the norm, we make the usual construction of a cusp form $\pi$ of $\mathrm{GL}\left(2, \boldsymbol{A}_{Q}\right)$ (see, e.g., the discussion in [3], $\S 7 \mathrm{~B}$ ). The central character of $\pi$ will be the product of the grössencharakter of $\boldsymbol{Q}$ associated to the extension $K / Q$, and the restriction of $\psi$ to $I_{Q}$, i.e., the grössencharakter associated to $\boldsymbol{Q}(\sqrt{3}) / \boldsymbol{Q}$. It is easy to check that this product is the grössencharakter associated to the extension $E=\boldsymbol{Q}(\sqrt{-6})$ of $\boldsymbol{Q}$.

Now consider the lifting $\Pi$ of $\pi$ to $\mathrm{GL}\left(2, \boldsymbol{A}_{E}\right)$. Its central character, the composition of the central character of $\pi$ with the norm $N: I_{E} \rightarrow I_{Q}$, is trivial. Moreover, $\Pi$ is cuspidal. Indeed the only way $\Pi$ could fail to be cuspidal would be for $\pi$ to be associated to a grössencharakter of $E$ (see [4], Theorem 2). But we have calculated the Euler factor for $p=19$ to be $\left(1+i p^{-s}\right)^{2}$, which could not come from a grössencharakter of $E$, since $p=19$ does not split in $E$. 
Thus $\pi$ and $\Pi$ are both cuspidal, $\Pi$ is trivial on the center, $\pi$ is not. So $\Pi$ gives rise to a representation $\bar{\Pi}$ of $\operatorname{PGL}\left(2, \boldsymbol{A}_{E}\right)$, which is the example we sought. It is Galois invariant but not a lifting.

\section{REFERENCES}

1. A. Borel, Automorphic L-functions, Proc. Symp. Pure Math., 33 (1979), part 2, $27-61$.

2. L. Clozel, "Base Change" géométrique: Relèvement de la série principale de GL(n, $\boldsymbol{C} / \boldsymbol{R})$, Actes du Colloque d'Analyse Harmonique Non Commutative, Marseille-Luminy, Lect. Notes Math., Vol. 728.

3. S. Gelbart, Automorphic forms on Adele groups, Annals of Math. Studies, \#83 (1975).

4. P. Gérardin and J. P. Labesse, The solution of the base change problem for GL(2), Proc. Symp. Pure Math., 33 (1979), part 2, 115-133.

5. R. P. Langlands, On the Classification of Irreducible Representations of Real Algebraic Groups, Notes, Institute for Advanced Study, 1973.

6. - Base Change for GL(2): the theory of Saito-Shintani with applications, Notes, Institute for Advanced Study, 1975.

7. J. Repka, Base change for tempered irreducible representations of $\mathrm{GL}(n, \boldsymbol{R})$, Pacific J. Math., 92 (1981).

8. T. Shintani, On irreducible unitary characters of a certain group extension of GL(2, C), J. Math. Soc. Japan, 29 (1977), 165-188.

Received February 29, 1980.

UNIVERSITY OF TORONTO

TORONTO, CANADA 


\section{PACIFIC JOURNAL OF MATHEMATICS}

\section{EDITORS}

DONALD BABBITT (Managing Editor)

University of Galifornia

Los Angeles, California 90024

Hugo RossI

University of Utah

Salt Lake City, UT 84112

C. C. MOORE AND ANDREW OGG

University of California

Berkeley, CA 94720
J. DugundJI

Department of Mathematics University of Southern California Los Angeles, California 90007

R. FinN and J. Milgram Stanford University Stanford, California 94305

\section{ASSOCIATE EDITORS}

R. ARENS

E. F. BeCKenbaCh

B. H. NEUManN

F. WOLF

K. YosHIDA

\section{SUPPORTING INSTITUTIONS}

UNIVERSITY OF ARIZONA

UNIVERSITY OF BRITISH COLUMBIA

CALIFORNIA INSTITUTE OF TECHNOLOGY

UNIVERSITY OF CALIFORNIA

MONTANA STATE UNIVERSITY

UNIVERSITY OF NEVADA, RENO

NEW MEXICO STATE UNIVERSITY

OREGON STATE UNIVERSITY
UNIVERSITY OF OREGON

UNIVERSITY OF SOUTHERN CALIFONIA

STANFORD UNIVERSITY

UNIVERSITY OF HAWAII

UNIVERSITY OF TOKYO

UNIVERSITY OF UTAH

WASHINGTON STATE UNIVERSITY

UNIVERSITY OF WASHINGTON 


\section{Pacific Journal of Mathematics}

\section{Vol. 95, No. $1 \quad$ September, 1981}

John Allen Beachy and William David Blair, On rings with bounded

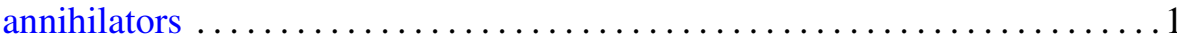

Douglas S. Bridges, A constructive look at positive linear functionals on

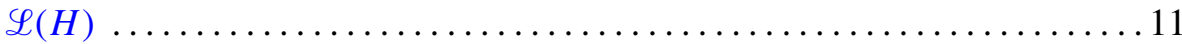

Muneo Chō and Makoto Takaguchi, Boundary points of joint numerical ranges

W. J. Cramer and William O. Ray, Solvability of nonlinear operator equations

Lester Eli Dubins and Gideon Schwarz, Equidiscontinuity of

Borsuk-Ulam functions

Maria Fragoulopoulou, Spaces of representations and enveloping 1.m.c.

*-algebras

Robert F. Geitz and J. Jerry Uhl, Jr., Vector-valued functions as families of scalar-valued functions

Ross Geoghegan, The homomorphism on fundamental group induced by a homotopy idempotent having essential fixed points

Ross Geoghegan, Splitting homotopy idempotents which have essential fixed points

Paul Jacob Koosis, Entire functions of exponential type as multipliers for

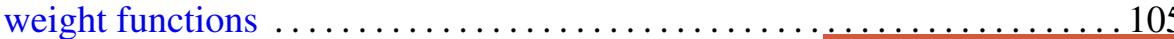

David London, Monotonicity of permanents of certain doubly stochastic matrices

Howard J. Marcum, Two results on cofibers

Giancarlo Mauceri, Zonal multipliers on the Heisenberg group

Edward Wilfred Odell, Jr. and Y. Sternfeld, A fixed point theorem in $c_{0} \quad \ldots 161$

Bernt Karsten Oksendal, Brownian motion and sets of harmonic measure zero

Andrew Douglas Pollington, The Hausdorff dimension of a set of normal numbers

Joe Repka, Base change lifting and Galois invariance ...

Gerald Suchan, Concerning the minimum of permanents on doubly stochastic circulants

Jun-ichi Tanaka, On isometries of Hardy spaces on compact abelian groups

Aaron R. Todd, Quasiregular, pseudocomplete, and Baire spaces 